\title{
Propiedades Psicométricas de la Versión Reducida de la Escala de Mitos hacia el Amor en Estudiantes de El Salvador
}

\section{Psychometric Properties of the Reduced Version of the Myths Scale Toward Love in Salvadoran Students}

\author{
Enrique Bonilla-Algovia y Esther Rivas Rivero \\ Área de Psicología Social, Facultad de Ciencias de la Educación, Universidad de Alcalá
}

\begin{abstract}
El objetivo de la investigación fue analizar las propiedades psicométricas de la Escala de Mitos hacia el Amor en una muestra de estudiantes de El Salvador y conocer si existen diferencias entre hombres y mujeres en la interiorización de los mitos del amor romántico. La muestra estuvo formada por 211 estudiantes (38,4\% hombres y $61,6 \%$ mujeres) de 2 universidades de San Salvador y Sonsonate, con una edad media de 22,43 años $(D E=4,92)$. Los resultados del análisis factorial exploratorio y del análisis factorial confirmatorio reportaron una estructura de 2 factores. La confiabilidad de la escala, evaluada con alfa de Cronbach, el coeficiente $\mathrm{H}$ y la fiabilidad compuesta, fue aceptable. Los mitos del amor romántico correlacionaron con la Escala de Sexismo Ambivalente. Los hombres, en comparación con las mujeres, están más de acuerdo con la creencia de que se puede amar a quien se maltrata. El instrumento puede ser útil para analizar los mitos del amor romántico en El Salvador.
\end{abstract}

Palabras clave: amor romántico, mitos, sexismo, validez

The aims of the study were to analyze the psychometric properties of the Myths Scale Toward Love in a sample of Salvadoran students and determine if there are any differences between men's and women's adoption of the myths of romantic love. The sample comprised 211 students ( $38.4 \%$ men and $61.6 \%$ women) from 2 universities located in San Salvador and Sonsonate, whose mean age was 22.43 years $(S D=4.92)$. The results of the exploratory factor analysis and the confirmatory factor analysis indicated a 2 -factor structure. The reliability of the scale, evaluated with Cronbach's alpha, the index H, and composite reliability, was acceptable. Romantic love myths were found to correlate with the Ambivalent Sexism Inventory. Compared to women, men agree more with the belief that one can love someone who one mistreats. The instrument can be useful for analyzing the myths of romantic love in El Salvador.

Keywords: romantic love, myths, sexism, validity

La violencia contra las mujeres es un problema social y una de las expresiones más extremas de la desigualdad de género y del sistema patriarcal (Ferrer Pérez \& Bosch Fiol, 2006; McCarthy et al., 2018; Oblitas Béjar, 2009). Entre las diferentes formas que puede adoptar la violencia contra las mujeres, la que tiene lugar en el ámbito de las relaciones de parejas heterosexuales es una de las más frecuentes (Aiquipa Tello, 2015; Ruiz-Pérez et al., 2004). Esta suele clasificarse en violencia sexual, física, psicológica y económica. En todos los contextos geográficos la violencia contra las mujeres en la pareja es un problema frecuente; sin embargo, los datos de prevalencia varían en función del país (García-Moreno et al., 2006; Guedes et al., 2014). En un estudio multipaís, realizado con una muestra de 24.097 mujeres de 15 a 49 años de distintos entornos culturales, se encontró que la prevalencia de violencia física y/o sexual a manos de una pareja masculina osciló entre el 15 y el 71\%, dejando ver que la violencia contra las mujeres está presente en todo el mundo (García-Moreno et al., 2006). En América Latina y el Caribe, el porcentaje de mujeres alguna vez casadas o unidas que han sido objeto de violencia física o sexual por parte de la pareja oscila entre el 17 y el 53,3\% (Bott et al., 2014). Por tanto, se trata de un problema de salud pública que tiene importantes repercusiones, tanto en la salud de las mujeres como en la sociedad en su conjunto

Enrique Bonilla-Algovia (D) https://orcid.org/0000-0003-1667-1880

Esther Rivas Rivero (iD https://orcid.org/0000-0001-5092-1260

El trabajo se ha realizado con el apoyo de un Contrato Predoctoral de Formación de Profesorado Universitario de la Universidad de Alcalá.

La correspondencia relativa a este artículo debe ser dirigida a Enrique Bonilla-Algovia, Facultad de Ciencias de la Educación, Universidad de Alcalá, Calle San Cirilo s/n, 28801, Alcalá de Henares, Madrid, España. Email: enrique.bonilla@uah.es 
(Bonilla-Algovia et al., 2020; Delgado-Álvarez et al., 2012; Kramer et al., 2004; Puente-Martínez et al., 2016; Rivas-Rivero \& Bonilla-Algovia, 2020).

En El Salvador, contexto en el que tuvo lugar esta investigación, la violencia contra las mujeres y los feminicidios son dos de los problemas más graves a los que se enfrenta el país (Navarro-Mantas \& Velásquez, 2016). Sierra et al. (2009), en un estudio realizado con una muestra de 640 mujeres salvadoreñas emparejadas, hallaron que aproximadamente el $20 \%$ se encontraba sufriendo abuso físico y el $30 \%$ abuso no físico por parte de la pareja. La Organización Panamericana de la Salud reporta que en El Salvador el 44,2\% de las mujeres de 15 a 49 años ha sido objeto de violencia emocional y el $26,3 \%$, de violencia física o sexual por parte de la pareja en algún momento de su vida (Bott et al., 2014). Un estudio más reciente, con una muestra representativa de mujeres salvadoreñas con edades entre los 15 y los 64 años, encontró que más de la mitad de las que han tenido una pareja ha sufrido algún tipo de violencia (física, psicológica o sexual) a manos de su pareja o expareja durante su vida (Navarro-Mantas et al., 2018).

Ante la alta tasa de prevalencia de violencia contra las mujeres en la pareja, se hace imprescindible analizar todos los elementos que podrían relacionarse con esta. Las creencias y actitudes son factores que pueden influir en la aparición de distintas conductas violentas en las relaciones íntimas (García-Cueto et al., 2015). Determinadas creencias sobre el amor pueden convertirse en el trasfondo de la violencia contra las mujeres (Caro Blanco, 2008). Los modelos tradicionales de amor romántico están basados en la jerarquía patriarcal y en el sistema sexo-género (García Villanueva et al., 2019) y se transmiten a través de la socialización. El amor romántico se articula en torno a una serie de mitos que lo vinculan con la abnegación, la dependencia, la complementariedad, la posesión, el sufrimiento, los celos, el control, entre otros. Los mitos del amor romántico pueden ser definidos como el conjunto de creencias compartidas a nivel social acerca de la supuesta naturaleza del amor (Yela García, 2000). Se trata de creencias expresadas como verdades absolutas y poco flexibles que se asientan sobre la desigualdad de género (Bosch Fiol et al., 2008; Ferrer et al., 2010). Los mitos románticos chocan con el ejercicio de la libertad individual (Caro Blanco, 2008) y generan algunas de las condiciones que posibilitan la aparición de violencia contra las mujeres en la pareja (Cubells Serra \& Calsamiglia Madurga, 2015). En la adolescencia y juventud, además, determinadas situaciones de maltrato son interpretadas como muestras de amor, lo que dificulta la identificación de la violencia en el noviazgo (García-Díaz et al., 2018).

El sexismo ambivalente es un constructo que está formado por dos dimensiones: el sexismo hostil y el sexismo benévolo (Glick \& Fiske, 1996). El primero coincide con el sexismo tradicional y se refiere a las actitudes negativas y prejuiciosas basadas en la supuesta inferioridad de las mujeres, mientras que el segundo hace referencia a un conjunto de actitudes que, ocultas tras un tono afectivo aparentemente positivo, encasillan a las mujeres en ciertos roles y mandatos de género. Ambos tipos de sexismo funcionan como un sistema de actitudes que mantiene la desigualdad estructural entre hombres y mujeres (Connelly \& Heesacker, 2012; Hammond \& Sibley, 2011).

La literatura científica reporta una vinculación entre el sexismo ambivalente y los mitos del amor romántico, de manera que una mayor aceptación del sexismo está unida a una mayor aceptación del amor romántico y viceversa (Rodríguez-Castro et al., 2013). En Colombia, en un estudio con población universitaria se encontró que la Escala de Mitos hacia el Amor correlacionaba con la Escala de Sexismo Ambivalente (Bonilla Algovia \& Rivas Rivero, 2018). En España, en un estudio con población adolescente, se hallaron correlaciones entre la Escala de Mitos hacia el Amor, el sexismo hostil y el sexismo benévolo (Carrascosa et al., 2019). Debido a ello, las validaciones que se han realizado de la Escala de Mitos hacia el Amor en distintos países han utilizado las correlaciones de Pearson entre esta y la Escala de Sexismo Ambivalente como prueba de validez externa (Bonilla Algovia \& Rivas Rivero, 2018; Rodríguez-Castro et al., 2013).

A pesar de que los mitos del amor romántico han sido analizados en varios estudios en América Latina (Bonilla Algovia \& Rivas Rivero, 2018) y en España (Bosch Fiol et al., 2008; Ferrer et al., 2010; Ferrer Pérez et al., 2010; Rodríguez-Castro et al., 2013), no se han encontrado investigaciones sobre el tema en el contexto salvadoreño. Es por ello por lo que el objetivo de este trabajo fue analizar, en una muestra de estudiantes de El Salvador, las propiedades psicométricas de la versión reducida de la Escala de Mitos hacia el Amor. 


\section{Método}

\section{Participantes}

La muestra estuvo formada por 211 estudiantes (38,4\% hombres y $61,6 \%$ mujeres) de dos universidades privadas de San Salvador y Sonsonate. Los y las estudiantes fueron seleccionados mediante un muestreo no probabilístico intencional entre quienes cursaban licenciaturas en ciencias de la educación. Los hombres tenían una edad media de 23 años $(D E=5,35)$ y las mujeres de 22,08 años $(D E=4,63), t(209)=1,33$, $p=0,189$. El $50 \%$ de hombres y el $43,1 \%$ de mujeres tenía pareja en el momento en el que el estudio tuvo lugar, $\left.\chi^{2}(1, n=210)=0,96, p=0,328\right)$. En la Tabla 1 se muestran las características sociodemográficas de la muestra. Tras analizar los valores perdidos en la Escala de Mitos hacia el Amor, un participante fue eliminado por no contestar, quedando una muestra final de 210 participantes $(38,1 \%$ hombres y $61,9 \%$ mujeres).

\section{Tabla 1}

Características Sociodemográficas de las Personas Participantes

\begin{tabular}{lrrr}
\hline & $n$ & $\%$ & Media $(D E)$ \\
\hline Sexo & & & \\
$\quad$ Hombre & 81 & 38,4 & \\
$\quad$ Mujer & 130 & 61,6 & $22,43(4,92)$ \\
Edad media & & & \\
Situación sentimental & & & \\
$\quad$ Con pareja & 96 & 45,7 & \\
$\quad$ Sin pareja & 114 & 54,3 & \multirow{2}{*}{ (15,98 (2,72) } \\
Edad a la que se tuvo pareja por 1 ${ }^{\text {a }}$ vez & & & \\
Situación económica de la familia & & & \\
$\quad$ Trabaja uno de los progenitores & 86 & 41,6 & \\
$\quad$ Trabajan ambos progenitores & 99 & 47,8 & \\
$\quad$ No trabaja ninguno de los progenitores & 6 & 2,9 & \\
$\quad$ Trabajan otros familiares & 16 & 7,7 & \\
$\quad$
\end{tabular}

\section{Instrumentos}

El cuestionario quedó conformado por los siguientes apartados:

\section{Caracteristicas Sociodemográficas}

Incluyó sexo, edad, situación económica de la familia, situación sentimental y edad a la que se tuvo pareja por primera vez.

\section{Escala de Mitos hacia el Amor (Bosch Fiol et al., 2008)}

Se utilizó la versión reducida validada en España (Rodríguez-Castro et al., 2013) y Colombia (Bonilla Algovia \& Rivas Rivero, 2018). El objetivo de la escala es medir la aceptación de las creencias sobre el amor romántico. La versión reducida consta de siete ítems que se agrupan en dos factores: Idealización del Amor (5 ítem; e.g, El amor es ciego) y Vinculación Amor-Maltrato (2 ítems; e.g, Se puede amar a alguien a quien se maltrata). Los ítems tienen un formato de respuesta tipo Likert de cinco alternativas $(1=$ completamente en desacuerdo, $2=$ en desacuerdo, $3=$ ni de acuerdo ni desacuerdo, $4=$ de acuerdo y $5=$ completamente de acuerdo). Por lo tanto, a mayor puntuación en la escala mayor aceptación de los mitos del amor romántico. A pesar de que la escala esté compuesta por dos factores, permite analizar los mitos del amor romántico de forma individual. Para analizar las propiedades psicométricas de la versión reducida de la Escala de Mitos hacia el Amor en El Salvador, se decidió mantener la numeración original de los ítems (ver Tabla 2). 


\section{Escala de Sexismo Ambivalente (ASI; Glick \& Fiske, 1996)}

El objetivo de la escala es medir las actitudes sexistas ambivalentes que se dirigen hacia las mujeres. En la investigación se utilizó la versión reducida y validada en España (Rodríguez Castro et al., 2009) y en El Savador y México (Bonilla-Algovia \& Rivas-Rivero, en prensa). Esta versión está compuesta por 12 ítems que se agrupan en dos factores de seis ítems cada uno: Sexismo Hostil (e.g., Las mujeres intentan ganar poder controlando a los hombres) y Sexismo Benévolo (e.g, Todo hombre debe tener una mujer a quien amar). El formato de respuesta es tipo Likert de seis alternativas $(0=$ totalmente en desacuerdo, $1=$ moderadamente en desacuerdo, $2=$ levemente en desacuerdo, $3=$ levemente de acuerdo, $4=$ moderadamente de acuerdo y $5=$ totalmente de acuerdo). Un mayor valor representa mayor aceptación del sexismo. Para la muestra del presente estudio, alfa de Cronbach fue 0,82 en la escala total ( $\alpha$ en sexismo hostil $=$ 0,86 ; $\alpha$ en sexismo benévolo $=0,76$ ).

\section{Procedimiento}

Para solicitar la colaboración de las universidades, se acudió a las instituciones de forma presencial y se explicaron las características del estudio. Las universidades con las que se contactó accedieron a participar y permitieron el acceso a sus estudiantes. Posteriormente se informó a los y las estudiantes de los objetivos de la investigación. La participación fue estrictamente voluntaria y los datos se recogieron de forma anónima, asegurando la confidencialidad de la información. Las personas que decidieron participar no recibieron ninguna remuneración y tuvieron la opción de abandonar el estudio en todo momento. Antes de responder el cuestionario, se explicó el procedimiento y se solicitó un consentimiento informado. El cuestionario fue autoaplicado en las universidades y se respondió en un tiempo aproximado de 40 minutos.

\section{Análisis de Datos}

La base de datos se creó y analizó con el programa estadístico SPSS 22.0. Como estadísticos descriptivos se utilizaron la media, el error estándar de la media y la desviación estándar. Se realizó un análisis factorial exploratorio (AFE) y un análisis factorial confirmatorio (AFC). Este último se hizo con el programa estadístico AMOS 24.0. Antes de realizar los análisis factoriales, se contrastó la adecuación muestral mediante las pruebas de Kaiser-Meyer-Olkin (KMO) y de esfericidad de Bartlett. La normalidad multivariada se analizó a través del test de Mardia (1974). Los resultados del test reportaron un coeficiente de 13,62, por lo que la técnica de estimación utilizada en los análisis factoriales fue máxima verosimilitud (Rodríguez Ayán \& Ruiz Díaz, 2008). Para la realización del AFE, además de la extracción por máxima verosimilitud, se aplicaron los siguientes criterios: autovalores mayores que 1 y eliminación de coeficientes menores que 0,30. Las características de los diferentes modelos comparados en el AFC se analizaron mediante los siguientes indicadores de bondad de ajuste: $\chi^{2} / g l<3$; índice de bondad de ajuste de Jöreskog (GFI), índice ajustado de bondad de ajuste de Jöreskog (AGFI), índice normalizado de Bentler-Bonett (NFI), índice de ajuste incremental (IFI) e índice comparativo de Bentler-Bonett (CFI) > 0,90; y la raíz del error cuadrático medio de aproximación (RMSEA) y la raíz del residuo cuadrático medio estandarizado (RMSR) < 0,08. La confiabilidad de la escala se calculó mediante alfa de Cronbach, el coeficiente $\mathrm{H}$ y la fiabilidad compuesta, que tiene en cuenta las interrelaciones de los factores extraídos. Para analizar las diferencias entre hombres y mujeres se calculó $t$ de Student y el tamaño del efecto. La validez externa fue analizada mediante las correlaciones lineales de Pearson $(r)$ entre la Escala de Mitos hacia el Amor y la Escala de Sexismo Ambivalente.

\section{Resultados}

El coeficiente de la prueba KMO fue 0,638 y la esfericidad de Bartlett reportó un valor de $p$ estadísticamente significativo, $\chi^{2}=284,14, p<0,001$, de manera que se procedió a la realización del análisis de la estructura factorial. El AFE arrojó una estructura de dos factores con autovalores iniciales mayores que la unidad, con una varianza explicada de los mitos sobre el amor del 56,66\% entre ambos factores. En la Tabla 2 se pueden ver la matriz factorial obtenida en el AFE y los pesos factoriales de los ítems que forman la escala.

El Factor 1 se correspondería con el componente Vinculación Amor-Maltrato y el Factor 2 con el componente Idealización del Amor. Sin embargo, la distribución de uno de los ítems no se corresponde con 
la hallada en la validación española (Rodríguez-Castro et al., 2013) y en la validación colombiana (Bonilla Algovia \& Rivas Rivero, 2018). El ítem 6, que en los estudios citados pertenece a la subescala Idealización del Amor, en este trabajo forma parte de la subescala Vinculación Amor-Maltrato. Esta distribución también podría ser plausible teóricamente, ya que "los celos aparecen de forma sistemática cuando se aborda el tema de la violencia en la pareja" (Perles Novas et al., 2011, p. 36) y pueden utilizarse para justificar actos violentos en las relaciones íntimas (Yela, 2003). Por lo tanto, los dos modelos posibles se pusieron a prueba mediante un AFC. El modelo de la validación española y colombiana obtuvo un ajuste inadecuado, $\chi^{2}(13, n=210)=52,34, p<0,001 ; \chi^{2} / g l=4,026 ; \mathrm{GFI}=0,93 ; \mathrm{AGFI}=0,86 ; \mathrm{CFI}=0,85 ; \mathrm{NFI}=0,82$; IFI $=0,86 ;$ RMSEA $=0,12 ;$ RMSR $=0,10$. Sin embargo, el modelo hallado en el AFE reportó un buen ajuste en todos los indicadores, $\chi^{2}(13, n=210)=23,55, p=0,036 ; \chi^{2} / g l=1,81$; GFI = 0,97; AGFI = 0,93; CFI = 0,96; NFI = 0,$92 ;$ IFI $=0,96 ;$ RMSEA $=0,06 ;$ RMSR $=0,06$.

\section{Tabla 2}

\section{Matriz Factorial}

\begin{tabular}{lcc}
\hline \multicolumn{1}{c}{ Ítem } & Factor 1 & Factor 2 \\
\hline 1. En alguna parte hay alguien predestinado para cada persona ("tu media naranja") & 0,64 \\
2. La pasión intensa de los primeros tiempos de una relación debería durar siempre & 0,59 \\
3. El amor es ciego & 0,32 \\
10. El amor verdadero lo puede todo & 0,66 \\
6. Los celos son una prueba de amor & 0,38 \\
8. Se puede amar a alguien a quien se maltrata & 0,70 \\
9. Se puede maltratar a alguien a quien se ama & 0,83 \\
\hline
\end{tabular}

$n=210$.

En la Tabla 3 se muestran los índices de consistencia interna de las subescalas y los descriptivos de cada uno de los ítems de la escala. Los pesos de regresión estandarizados obtenidos en el AFC oscilan entre 0,27 y 0,84 y son significativos en los siete ítems $(p<0,05)$. Esta significación indica que todas las variables están relacionadas con el constructo en el que han sido incluidas. En este sentido, el modelo quedaría compuesto del siguiente modo: factor Idealización del Amor (ítems 1, 2, 3 y 10) y factor Vinculación AmorMaltrato (ítems 6, 8 y 9). La confiabilidad del constructo puede considerarse aceptable. Los mitos sobre el amor que cuentan con una mayor aceptación son los que pertenecen a la idealización del amor, con puntuaciones medias superiores a las obtenidas en los mitos de la vinculación amor-maltrato. Los mitos más aceptados son el de la pasión eterna (ítem 2) y el de la omnipotencia (ítem 10), mientras que los mitos más rechazados son los de la ambivalencia (ítems 8 y 9).

Tabla 3

Propiedades Psicométricas de los Ítems y de los Factores

\begin{tabular}{lccccccc}
\hline \multicolumn{1}{c}{ Ítem } & $R$ & $M$ & $E E$ & $D E$ & $\alpha$ & $\mathrm{CR}$ & $\mathrm{H}$ \\
\hline Ítem 1 & 0,71 & 3,64 & 0,09 & 1,30 & & & \\
Ítem 2 & 0,63 & 4,05 & 0,08 & 1,15 & 0,65 & 0,68 & 0,73 \\
Ítem 3 & 0,27 & 3,35 & 0,09 & 1,36 & & & \\
Ítem 10 & 0,70 & 3,70 & 0,09 & 1,33 & & & \\
Ítem 6 & 0,42 & 2,09 & 0,08 & 1,13 & & \multirow{2}{*}{0,71} & 0,79 \\
Ítem 8 & 0,74 & 1,67 & 0,06 & 0,86 & 0,67 & & \\
Ítem 9 & 0,84 & 1,58 & 0,07 & 0,99 & & & \\
\hline
\end{tabular}

Nota. $n=210 . R=$ pesos de regresión estandarizados; $M=$ media; $E E=$ error estándar de la media; $D E=$ desviación estándar; $\alpha=$ alfa de Cronbach; $\mathrm{CR}=$ fiabilidad compuesta $; \mathrm{H}=$ coeficiente $\mathrm{H}$. 
Las correlaciones entre los ítems de la misma subescala son directas (véase la Tabla 4). La correlación más alta es la que se da entre los ítems 8 y $9(r=0,61, p<0,001)$, que pertenecen al factor Vinculación Amor-Maltrato, mientras que la más baja es la que se da entre los ítems 8 y 10 ( $r=0,00, p=0,999)$. Los resultados de las correlaciones lineales de Pearson entre la Escala de Mitos hacia el Amor y la Escala de Sexismo Ambivalente reportan una asociación directa entre ambas escalas $(r=0,40, p<0,001)$, de manera que tanto el sexismo hostil $(r=0,23, p<0,001)$ como el sexismo benévolo $(r=0,41, p<0,001)$ presentan correlaciones directas con los mitos del amor romántico. Por último, el coeficiente de correlación de Pearson entre los factores Idealización del Amor y Vinculación Amor-Maltrato es 0,17 $(p=0,810)$.

\section{Tabla 4}

Correlaciones entre los Ítems

\begin{tabular}{lccccccc}
\hline & Ítem 1 & Ítem 2 & Ítem 3 & Ítem 6 & Ítem 8 & Ítem 9 & Ítem 10 \\
\hline Ítem 1 & - & & & & & & \\
Ítem 2 & $0,44^{* *}$ & - & & & & & \\
Ítem 3 & $0,17^{* *}$ & $0,20^{* *}$ & - & & & & \\
Ítem 6 & 0,02 & 0,07 & $0,22^{* *}$ & - & & & \\
Ítem 8 & $-0,08$ & $-0,08$ & 0,02 & $0,32^{* *}$ & - & & \\
Ítem 9 & $-0,09$ & $-0,08$ & 0,10 & $0,35^{* *}$ & $0,61^{* *}$ & - & \\
Ítem 10 & $0,50^{* *}$ & $0,44^{* *}$ & $0,19^{* *}$ & 0,06 & 0,00 & $-0,13$ & - \\
\hline \multicolumn{2}{l}{ Nota. $n=210 .{ }^{*} p \leq 0,05 ; * * p \leq 0,01$}
\end{tabular}

En la Tabla 5 se exponen las diferentes puntuaciones obtenidas por hombres y mujeres en el acuerdo con los mitos del amor romántico. En la puntuación media de la Escala de Mitos hacia el Amor, el grado de acuerdo de hombres y mujeres es muy similar ( $\left.M_{\text {hombres }}=2,88, D E=0,62 ; M_{\text {mujeres }}=2,86, D E=0,62\right)$, $t(208)=0,26, p=0,795$. Sin embargo, al realizar el análisis individual de los ítems, los hombres puntúan más alto que las mujeres en el ítem 8 , "se puede amar a alguien a quien se maltrata".

Tabla 5

Diferencias entre Mujeres y Hombres en la Aceptación de los Mitos

\begin{tabular}{|c|c|c|c|c|c|c|c|}
\hline & \multicolumn{2}{|c|}{ Hombres } & \multicolumn{2}{|c|}{ Mujeres } & \multirow{2}{*}{$t$} & \multirow{2}{*}{$p$} & \multirow{2}{*}{$d$} \\
\hline & $M$ & $D E$ & $M$ & $D E$ & & & \\
\hline Ítem 1 & 3,64 & 1,31 & 3,65 & 1,29 & $-0,05$ & 0,963 & \\
\hline Ítem 2 & 3,89 & 1,30 & 4,15 & 1,04 & $-1,51$ & 0,134 & \\
\hline Ítem 3 & 3,33 & 1,34 & 3,36 & 1,37 & $-0,19$ & 0,850 & \\
\hline Ítem 10 & 3,61 & 1,29 & 3,75 & 1,36 & $-0,71$ & 0,481 & \\
\hline Ítem 6 & 2,14 & 1,08 & 2,06 & 1,17 & 0,47 & 0,638 & \\
\hline Ítem 8 & 1,88 & 0,92 & 1,55 & 0,80 & 2,64 & 0,009 & 0,39 \\
\hline Ítem 9 & 1,70 & 1,05 & 1,51 & 0,95 & 1,37 & 0,173 & \\
\hline
\end{tabular}

Nota. $n$ (hombres) $=80, n$ (mujeres) $=130 ; t=t$ de Student; $d=$ tamaño del efecto $(d$ de Cohen).

\section{Discusión}

Los resultados del AFE arrojaron una estructura de dos factores; sin embargo, a diferencia de lo hallado en otros países (Bonilla Algovia \& Rivas Rivero, 2018; Rodríguez-Castro et al., 2013), el ítem 6 formaba parte del factor Vinculación Amor-Maltrato y no del factor Idealización del Amor. Esta distribución de los ítems también parece adecuada teóricamente, debido a que los celos, cuando son vistos como una muestra de amor, pueden servir como justificación de prácticas represivas e incluso violentas en las relaciones íntimas (Yela, 2003) y suelen aparecer en los estudios que abordan la violencia en la pareja (Perles Novas et 
al., 2011). En este sentido, en el contexto de la violencia contra las mujeres, tanto en la adolescencia como en la adultez los celos están íntimamente relacionados con el abuso por parte de la pareja y son un factor de riesgo para su aparición (Nóblega Mayorga, 2012; Rodríguez-Domínguez et al., 2018). Incluso en ausencia de violencia explícita, los celos han de ser considerados una forma de violencia implícita, puesto que son una estrategia de control y aislamiento de la pareja (Bosch-Fiol \& Ferrer-Pérez, 2012; Ferrer Pérez et al., 2010). Especialmente en los contextos con altas tasas de violencia contra las mujeres, como es el caso de El Salvador (Bott et al., 2014; Navarro-Mantas et al., 2018; Navarro-Mantas \& Velásquez, 2016; Sierra et al., 2009), la interpretación de los celos como una muestra de amor podría ser una forma de justificar los malos tratos, lo que explicaría que el ítem 6 ("los celos son una prueba de amor") se enmarque en el factor Vinculación Amor-Maltrato.

Las medidas de calidad de ajuste del AFC apoyaron la validez de la estructura hallada en el AFE y los pesos de regresión estandarizados fueron significativos en todos los ítems. Los coeficientes alfa de Cronbach obtenidos en cada una de las subescalas fueron superiores a los de la validación original en España (Bosch Fiol et al., 2008). Los coeficientes $\mathrm{H}$ fueron aceptables en ambas subescalas. La validez externa fue contrastada favorablemente mediante las correlaciones de Pearson entre la Escala de Mitos hacia el Amor y la Escala de Sexismo Ambivalente. Por lo tanto, la dimensionalidad del instrumento en la presente muestra ha quedado conformada de la siguiente manera: subescala Idealización del amor (ítems 1, 2, 3 y 10) y subescala Vinculación amor-maltrato (ítems 6, 8 y 9).

La correlación entre el sexismo ambivalente y los mitos del amor romántico coincide con lo obtenido en otras investigaciones, por lo que, en la muestra de estudiantes de El Salvador, una mayor interiorización del amor romántico está unida a una mayor interiorización del sexismo. En la misma línea que Carrascosa et al. (2019), los mitos románticos correlacionan en mayor medida con el sexismo benévolo que con el sexismo hostil. La explicación podría estar en que el sexismo benévolo se basa en la complementariedad de los roles tradicionales de género en las relaciones íntimas heterosexuales (Connelly \& Heesacker, 2012; Glick \& Fiske, 1996; Hammond \& Sibley, 2011), del mismo modo que los mitos románticos estructuran las relaciones íntimas en torno a la heteronormatividad y la complementariedad. El sexismo ambivalente y el amor romántico estarían relacionados y fomentarían la desigualdad de género en el plano relacional.

Los resultados hallados en otros países muestran diferencias entre hombres y mujeres respecto a la aceptación de varios mitos del amor romántico (Bonilla Algovia \& Rivas Rivero, 2018; Bosch Fiol et al., 2008; Ferrer et al., 2010; Rodríguez-Castro et al., 2013). En la presente investigación, en cambio, solo hay diferencias en uno de los mitos del amor-maltrato. Los hombres están más de acuerdo que las mujeres respecto a que "se puede amar a alguien a quien se maltrata". En España y Colombia también se encontró que los hombres estaban más de acuerdo con este mito (Bonilla Algovia \& Rivas Rivero, 2018; RodríguezCastro et al., 2013). Este tipo de creencias que vinculan el amor y la violencia son un factor de riesgo de la violencia contra las mujeres en la pareja (Caro Blanco, 2008; Cubells Serra \& Calsamiglia Madurga, 2015). Por lo tanto, en el trasfondo del amor romántico estarían la posesión y el abuso (García Villanueva et al., 2019). Teniendo en cuenta estos resultados, es necesario seguir profundizando en el estudio de la relación entre la violencia contra las mujeres y las concepciones sobre el amor romántico.

La ley especial integral para una vida libre de violencia para las mujeres, aprobada en El Salvador el 25 de noviembre de 2010 y publicada en enero de 2011, incluye la necesidad de prevenir la violencia desde el ámbito educativo, por lo que no se deben escatimar esfuerzos. La ideología del amor romántico es una construcción social, de manera que las creencias y los mitos románticos pueden ser modificados mediante la sensibilización y la intervención educativas. Existen evidencias de que los programas de prevención que han abordado la violencia contra las mujeres en El Salvador han obtenido buenos resultados en cuanto al reconocimiento de las situaciones violentas (Navarro-Mantas, 2013; Navarro-Mantas \& Velásquez, 2016). Por lo tanto, en el sistema educativo hay que seguir desarrollando acciones preventivas y de concienciación social (Racionero-Plaza et al., 2018), prestando especial atención a las creencias y normas sociales que podrían legitimar la desigualdad y la violencia

La principal limitación del estudio se encuentra en el criterio muestral empleado: la muestra está formada únicamente por estudiantes universitarios de ciencias de la educación y no es representativa de la población general; sin embargo, el tamaño muestral es adecuado para aplicar este tipo de análisis (Barrett, 2007). El hecho de que haya un mayor porcentaje de mujeres que de hombres es otro elemento para tener en cuenta. Hay que señalar, también, que la información se ha obtenido mediante una metodología transversal que no permite conocer la evolución de las creencias sobre el amor. En el futuro sería 
importante comprobar si la distribución de los ítems se mantiene en muestras más amplias o si, por el contrario, la estructura hallada en otros países presenta mejor calidad de ajuste. La significación de las comparaciones de medias entre mujeres y hombres puede haberse visto afectada por el tamaño muestral. Por último, en próximas investigaciones sería interesante realizar un análisis comparativo de la aceptación de los mitos del amor romántico en distintos países de América Latina.

Los resultados muestran la utilidad del instrumento para analizar los mitos del amor romántico en El Salvador. El hecho de contar con un instrumento de estas características puede ser un aliciente para que futuros estudios profundicen en el estudio del amor romántico y sus vínculos con la violencia contra las mujeres en la pareja, ya que El Salvador cuenta con escasas investigaciones centradas en esta temática. Además, la información que se obtenga a través de la escala puede ser tenida en cuenta a la hora de implementar programas de intervención en el ámbito socioeducativo.

\section{Referencias}

Aiquipa Tello, J. J. (2015). Dependencia emocional en mujeres víctimas de violencia de pareja. Revista de Psicología Pontificia Universidad Católica del Perú, 33(2), 412-437. http://www.scielo.org.pe/pdf/psico/v33n2/a07v33n2.pdf

Barrett, P. (2007). Structural equation modelling: Adjudging model fit. Personality and Individual Differences, 42(5), 815-824. https://doi.org/10.1016/j.paid.2006.09.018

Bonilla Algovia, E. \& Rivas Rivero, E. (2018). Propiedades psicométricas de la versión reducida de la Escala de Mitos sobre el Amor en una muestra de estudiantes colombianos. Suma Psicológica, 25(2), 162-170. https://doi.org/10.14349/sumapsi.2018.v25.n2.8

Bonilla-Algovia, E. \& Rivas-Rivero, E. (en prensa). Validez de la versión reducida de la Escala de Sexismo Ambivalente en El Salvador y México. Revista Mexicana de Psicología.

Bonilla-Algovia, E., Rivas-Rivero, E. \& Vázquez, J. J. (2020). Impact of gender-based violence on psychological distress and happiness in León (Nicaragua). Health Care for Women International, 41(6), 673-689. https://doi.org/10.1080/07399332.2020.1764564

Bosch-Fiol, E. \& Ferrer-Pérez, V. A. (2012). Nuevo mapa de los mitos sobre la violencia de género en el siglo XXI. Psicothema, 24(4), 548-554. https://www.redalyc.org/pdf/727/72723959007.pdf

Bosch Fiol, E., Ferrer Pérez, V. A., García Buades, M. E., Ramis Palmer, M. C., Mas Tous, M. C., Navarro Guzmán, C. \& Torrens Espinosa, G. (2008). Del mito del amor romántico a la violencia contra las mujeres en la pareja: año 2004 - año 2007. Gobierno de España, Ministerio de Igualdad, Secretaría de Igualdad de Políticas de Igualdad, Instituto de la Mujer. https://sistemadif.jalisco.gob.mx/cepavi/Material_didactico/Noviazgo/Modelo\%20de\%20amor.pdf

Bott, S., Guedes, A., Goodwin, M. \& Adams Mendoza, J. (2014). Violencia contra las mujeres en América Latina y el Caribe: análisis comparativo de datos poblacionales de 12 países. Organización Panamericana de la Salud. http://repositorio.minedu.gob.pe/handle/123456789/4109

Caro Blanco, C. (2008). Un amor a tu medida. Estereotipos y violencia en las relaciones amorosas. Revista de Estudios de Juventud, 83, 213-228. http://www.injuve.es/sites/default/files/RJ83-14.pdf

Carrascosa, L., Cava, M. -J., Buelga, S. \& de Jesús, S. -N. (2019). Reduction of sexist attitudes, romantic myths, and aggressive behaviors in adolescents: Efficacy of the DARSI program. Psicothema, 31(22), 121-127. https://doi.org/10.7334/psicothema2018.245

Connelly, K. \& Heesacker, M. (2012). Why is benevolent sexism appealing?: Associations with system justification and life satisfaction. Psychology of Women Quarterly, 36(4), 432-443. https://doi.org/10.1177/0361684312456369

Cubells Serra, J. \& Calsamiglia Madurga, A. (2015). El repertorio del amor romántico y las condiciones de posibilidad para la violencia machista. Universitas Psychologica, 14(5), 1681-1694. https://doi.org/10.11144/Javeriana.upsy14-5.rarc

Delgado-Álvarez, M. C., Sánchez Gómez, M. C. \& Fernández-Dávila Jara, P. A. (2012). Atributos y estereotipos de género asociados al ciclo de la violencia contra la mujer. Universitas Psychologica, 11(3), 769-777. https://doi.org/10.11144/Javeriana.upsy11-3.aega

Ferrer, V. A., Bosch, E. \& Navarro, C. (2010). Los mitos románticos en España. Boletín de Psicología, 99, 7-31. https://www.uv.es/seoane/boletin/previos/N99-1.pdf

Ferrer Pérez, V. A. \& Bosch Fiol, E. (2006). Las actitudes sexistas de los maltratadores: el uso de medidas perceptuales como alternativa. Revista Iberoamericana de Diagnóstico y Evaluación/e Avaliação Psicológica, 2(22), 157-181. https://www.redalyc.org/pdf/4596/459645449009.pdf

Ferrer Pérez, V. A., Bosch Fiol, E., Navarro Guzmán, C. \& Ferreiro Basurto, V. (2010). El mito romántico de los celos y su aceptación en la sociedad española actual. Apuntes de Psicología, 28(3), 391-402. http://apuntesdepsicologia.es/index.php/revista/article/view/227/229

García-Cueto, E., Rodríguez-Díaz, F. J., Bringas-Molleda, C., López-Cepero, J., Paíno-Quesada, S. \& Rodríguez-Fanco, L. (2015). Development of the Gender Role Attitudes Scale (GRAS) amongst young Spanish people. International Journal of Clinical and Health Psychology, 15(1), 61-68. https://doi.org/10.1016/j.ijchp.2014.10.004

García-Díaz, V., Lana-Pérez, A., Fernández-Feito, A., Bringas-Molleda, C., Rodríguez-Franco, L. \& Rodríguez-Díaz, F. J. (2018). Actitudes sexistas y reconocimiento del maltrato en parejas jóvenes. Atención Primaria, 50(7), 398-405. https://doi.org/10.1016/j.aprim.2017.04.001

García-Moreno, C., Jansen, H. A. F. M., Ellsberg, M., Heise, L. \& Watts, C. H. (2006). Prevalence of intimate partner violence: Findings from the WHO multi-country study on women's health and domestic violence. The Lancet, 368(9543), $1260-1269$. https://doi.org/10.1016/S0140-6736(06)69523-8

García Villanueva, J., Hernández Ramírez, C. I. \& Monter Arismendi, N. S. (2019). Amor romántico entre estudiantes universitarios (hombres y mujeres), una mirada desde la perspectiva de género. Revista de Estudios de Género La Ventana, 6(49), $218-247$. https://doi.org/10.32870/lv.v6i49.6996

Glick, P. \& Fiske, S. T. (1996). The Ambivalent Sexism Inventory: Differentiating hostile and benevolent sexism. Journal of Personality and Social Psychology, 70(3), 491-512. https://doi.org/10.1037/0022-3514.70.3.491 
Guedes, A., García-Moreno, C. \& Bott, S. (2014). Violencia contra las mujeres en Latinoamérica y el Caribe: un problema de salud pública de proporciones epidémicas. Foreign Affairs Latinoamérica, 14(1), 41-48. https://www.paho.org/hq/dmdocuments/2014/Foreign-Affairs-2014-Guedes-et-al-Violencia-contra-las-mujeres-en-LAC.pdf

Hammond, M. D. \& Sibley, C. G. (2011). Why are benevolent sexists happier? Sex Roles, 65(5-6), Article 332. https://doi.org/10.1007/s11199-011-0017-2

Kramer, A., Lorenzon, D. \& Mueller, G. (2004). Prevalence of intimate partner violence and health implications for women using emergency departments and primary care clinics. Women's Health Issues, 14(1), 19-29. https://doi.org/10.1016/j.whi.2003.12.002

Ley especial integral para una vida libre de violencia para las mujeres, Decreto 520, Asamblea Legislativa de la República de El Salvador (2011)._ https://www.asamblea.gob.sv/sites/default/files/documents/decretos/325EF057-A460-4BCE-AF15620CB8AD57E5.pdf

Mardia, K. V. (1974). Applications of some measures of multivariate skewness and kurtosis in testing normality and robustness studies. Sankhyā: The Indian Journal of Statistics Series B, 36(2), 115-128. https://www.jstor.org/stable/25051892

McCarthy, K. J., Mehta, R. \& Haberland, N. A. (2018). Gender, power, and violence: A systematic review of measures and their association with male perpetration of IPV. PLoS ONE, 13(11), Artículo e0207091. https://doi.org/10.1371/journal.pone.0207091

Navarro-Mantas, L. (2013). Resultados obtenidos a largo plazo de un programa de prevención de violencia de género en El Salvador. Entorno Universidad Tecnológica de El Salvador, 54, 12-19. https://doi.org/10.5377/entorno.v0i54.6291

Navarro-Mantas, L. \& Velásquez, M. J. (2016). Herramientas para prevenir la violencia de género: implicaciones de un registro diario de situaciones de desigualdad de género. Acta Colombiana de Psicología, 19(2), 139-148. https://doi.org/10.14718/ACP.2016.19.2.7

Navarro-Mantas, L., Velásquez, M. J., de Lemus, S. \& Megías, J. L. (2018). Prevalence and sociodemographic predictors of intimate partner violence against women in El Salvador. Journal of Interpersonal Violence. Anticipo en línea de la publicación. https://doi.org/10.1177/0886260518779065

Nóblega Mayorga, M. (2012). Características de los agresores en la violencia hacia la pareja. Liberabit, 18(1), 59-67. http://www.scielo.org.pe/scielo.php?pid=S1729-48272012000100008\&script=sci arttext\&tlng=en

Oblitas Béjar, B. (2009). Machismo y violencia contra la mujer. Investigaciones Sociales Universidad Nacional Mayor de San Marcos, 13(23), 301-322. https://doi.org/10.15381/is.v13i23.7235

Perles Novas, F., San Martín García, J., Canto Ortiz, J. \& Moreno Jiménez, P. (2011). Inteligencia emocional, celos, tendencia al abuso y estrategias de resolución de conflicto en la pareja. Escritos de Psicología, 4(1), 34-43. https://doi.org/10.5231/psy.writ.2011.0605

Puente-Martínez, A., Ubillos-Landa, S., Echeburúa, E. \& Páez-Rovira, D. (2016). Factores de riesgo asociados a la violencia sufrida por la mujer en la pareja: una revisión de meta-análisis y estudios recientes. Anales de Psicología, 32(1), 295-306. https://doi.org/10.6018/analesps.32.1.189161

Racionero-Plaza, S., Ugalde-Lujambio, L., Puigvert, L. \& Aiello, E. (2018). Reconstruction of autobiographical memories of violent sexual-affective relationships through scientific reading on love: A psycho-educational intervention to prevent gender violence. Frontiers in Psychology, 9, Artículo 1996. https://doi.org/10.3389/fpsyg.2018.01996

Rivas-Rivero, E. \& Bonilla-Algovia, E. (2020). Salud mental y miedo a la separación en mujeres víctimas de violencia de pareja. Revista Iberoamericana de Psicología y Salud, 11(1), 54-67. https://doi.org/10.23923/j.rips.2020.01.035

Rodríguez Ayán, M. N. \& Ruiz Díaz, M. Á. (2008). Atenuación de la asimetría y de la curtosis de las puntuaciones observadas mediante transformaciones de variables: incidencia sobre la estructura factorial. Psicológica, 29(2), 205-227. https://www.redalyc.org/pdf/169/16929206.pdf

Rodríguez Castro, Y., Lameiras Fernández, M. \& Carrera Fernández, M. V. (2009). Validación de la versión reducida de las escalas ASI $\mathrm{y}$ AMI en una muestra de estudiantes españoles. Psicogente, 12(22), 284-295. https://dialnet.unirioja.es/servlet/articulo?codigo=3265018

Rodríguez-Castro, Y., Lameiras-Fernández, M., Carrera-Fernández, M. V. \& Vallejo-Medina, P. (2013). La fiabilidad y validez de la Escala de Mitos hacia el Amor: las creencias de los y las adolescentes. Revista de Psicología Social, 28(2), 157-168. https://doi.org/10.1174/021347413806196708

Rodríguez-Domínguez, C., Durán Segura, M. \& Martínez-Pecino, R. (2018). Ciberagresores en el noviazgo adolescente y su relación con la violencia psicológica, el sexismo y los celos. Health and Addictions, 18(1), 17-27. https://doi.org/10.21134/haaj.v18i1.329

Ruiz-Pérez, I., Blanco-Prieto, P. \& Vives-Cases, C. (2004). Violencia contra la mujer en la pareja: determinantes y respuestas sociosanitarias. Gaceta Sanitaria, 18(Suplemento 2), 4-12. https://doi.org/10.1157/13061990

Sierra, J. C., Ortega, V., Gutiérrez-Quintanilla, J. R., Bermúdez, M. P. \& Buela-Casal, G. (2009). Violencia de pareja en mujeres salvadoreñas: prevalencia y factores sociodemográficos de riesgo. Revista Argentina de Clínica Psicológica, 18(2), 115-123. https://www.redalyc.org/pdf/2819/281921792002.pdf

Yela, C. (2003). La otra cara del amor: mitos, paradojas y problemas. Encuentros en Psicología Social, 1(2), 263-267.

Yela García, C. (2000). El amor desde la psicología social: ni tan libres, ni tan racionales. Pirámide.

Fecha de recepción: Mayo de 2019.

Fecha de aceptación: Enero de 2020. 